\title{
Modern methods for surface wastewater treatment
}

\author{
Oleg Primin* and Galina Varyushina \\ Moscow State University of Civil Engineering, 26, Yaroslavskoye shosse, Moscow, 129337, Russia
}

\begin{abstract}
The article gives the characteristics of the system of removal and purification of surface wastewater in Moscow. One of the features of this system is the large-scale use of hydrographic network of the city as elements of the drainage network. Currently improvement of the ecological state of water objects is only possible if to reduce anthropogenic load on them and watersheds, to recover self-cleaning abilities of water bodies and the to protect groundwater from contamination. The article reveals results of research, and contains technological parameters for facilities of surface sewage water purification and recommendations to improve their operation. Article also gives technical solutions to upgrade operated wastewater treatment systems and to ensure the achievement of required standards of treatment of surface runoff when dilution in water. Along with proposals for the reconstruction and construction of wastewater treatment plants with the classical technology of surface water treatment, it is necessary to consider the principle of decentralization with the use of local treatment facilities.
\end{abstract}

\section{Introduction}

Centralized collection and treatment of surface runoff is an urgent environmental task. Studies have found that the surface runoff from the economically developed areas of urban settlements, organized diversionary and diffuse, is one of the main sources of man-made pollution and degradation of water bodies located in the city limits. A special feature of the organization of the system of removal and treatment of surface runoff in Moscow is the large-scale use of the hydrographic network of the city as elements of the drainage network.

Currently, it is positioned as a "collector-river network of the city", which covers up to $90 \%$ of Moscow in its old borders. At the same time, up to 600 million cubic meters of surface wastewater is discharged annually into Moscow water bodies. The drainage system of Moscow consists of:

- the city's drainage network, with a length of 7.4 thousand $\mathrm{km}$, which houses 110,000 surface wastewater wells and 160,000 observation wells;

- water bodies-small rivers and water bodies in the amount of 211 units with a total area of 690 hectares and a length of open riverbeds and streams about $250 \mathrm{~km}$;

- A waterworks on the Yauza river, which provides flooding of the Moskva river;

${ }^{*}$ Corresponding author: tepper2007@yandex.ru 
- 13 snow-melting points for utilization of snow mass during winter cleaning of city streets and highways.

\section{Features of technologies for surface wastewater treatment}

Currently, the conditions of urban drainage networks of Moscow differ significantly from the design conditions adopted in its design. The weather conditions and intensity of precipitation have changed, respectively, the volume of surface wastewater has increased (PSV), with the implementation of reconstruction of the road surface, sidewalks, increased the number of waterproof surfaces (asphalt pavement highways, driveways, Parking lots, blocks of high-rise residential and office buildings, shopping and entertainment complexes), and the number of permeable surfaces (lawns, parks, squares, floodplains and nature reserves) has decreased dramatically.

For these reasons, the concept of total equipping with water treatment facilities of the drainage system, contained in the General scheme for the removal of surface runoff from the territory of Moscow in 2001 needs to be updated on the basis of real data on the state of the Moscow river and the feasibility study of this approach. [1,2]. One of the most important tasks in updating the "General scheme of drainage and treatment of surface runoff" in Moscow is a reasonable choice of surface wastewater treatment technology. We are talking about the development of engineering and technological solutions and the feasibility study for the construction of new and reconstruction of existing facilities (Figure 1) for the treatment and disinfection of surface wastewater entering the water bodies of Moscow from the territories of various functional purposes.

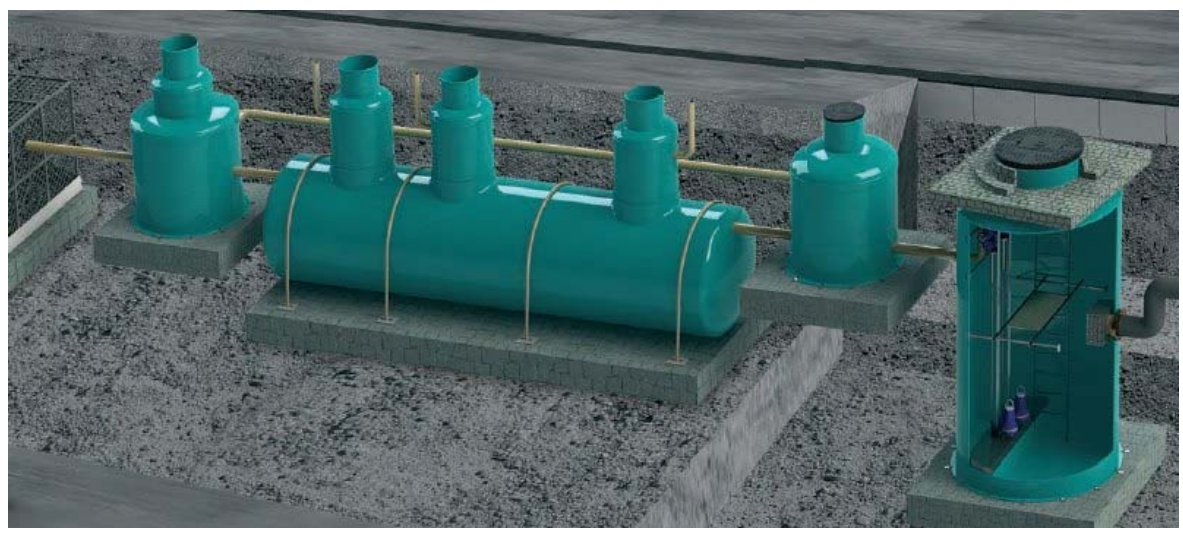

Fig. 1. Treatment facility "Standartpark".

Table 1. The effect of surface wastewater treatment on the main pollution indicators at the existing treatment facilities of the city.

\begin{tabular}{|c|c|c|c|c|}
\hline \multirow{2}{*}{ Treatment facilities } & \multicolumn{2}{|c|}{ Suspended solids } & \multicolumn{2}{c|}{ Petrochemicals } \\
\cline { 2 - 5 } & Out [mg/l] & Eff. [\%] & Out [mg/l] & Eff. [\%] \\
\hline Mechanical treatment & $10-20$ & $20-40$ & 3,0 & $10-15$ \\
\hline Chamber treatm,ent facilities & $10-20$ & $30-70$ & 3,0 & $10-15$ \\
\hline Thin-layer facilities & $30-40$ & $40-60$ & 10 & 60 \\
\hline Gabion filtration treatment facilities & $50-100$ & $10-20$ & 20 & $5-10$ \\
\hline Group facilities with reagents & $5-8$ & $80-90$ & $0,5-1,0$ & $80-90$ \\
\hline Tertiary treatment facilities with sorbtion & $1-5$ & $90-95$ & $0,03-0,1$ & $90-95$ \\
\hline
\end{tabular}


There is an advanced technology of disposal of surface runoff (PSV) for residential and industrial areas of the city developed in Moscow state university of civil engineering. Key indicators of the composition of the purified water meet the requirements of water discharge to fishery water bodies [3].

On the basis of researches on optimization of technological scheme of cleaning of surface wastewater and definitions of parameters of their work, recommendations on design which main content consisted in the following were developed: to collecting, averaging and cleaning all volume of the rain, melt and water-washing waters falling out on the territory of the catchment area is exposed. The calculation of the amount of surface wastewater runoff from 1 hectare of catchment surface is determined by the results of observations of hydrometeorological service in the area, which monitors the quantitative parameters of precipitation.

\section{Main solutions}

Wastewater is collected in sewers and enters the underground storage capacity, consisting of rectangular sections, the filling of which takes place through the overflow window, located in the upper part of the section. The first section of the storage tank is used as a grit to remove sedimentary impurities and small debris washed away from the catchment area. To ensure the gravity of the removal of surface wastewater from the catchment area treatment facilities are located in its lowest place. At the first stage of mechanical treatment the waste water passes through the forty-holding grate located in the well on the supply collector of PSV and enters the storage tank for sedimentation of effluents, while the excess volume of surface wastewater is poured from the last section through a special device into the city collector or pond. The design of the water transfer device eliminates the possibility of discharge into the reservoir of oil film, precipitate and other floating impurities. Figure 2 shows the room of pressure filters of facilities for treatment of wastewater from bus washing, located in the Northern administrative district of the Moscow. Mechanical cleaning of the surface wastewater by settling is carried out within 2 to 3 days, after which the clarified drains are directed to physical and chemical cleaning, including a cascade of three series-connected pressure filters with granular filtering and sorption materials.

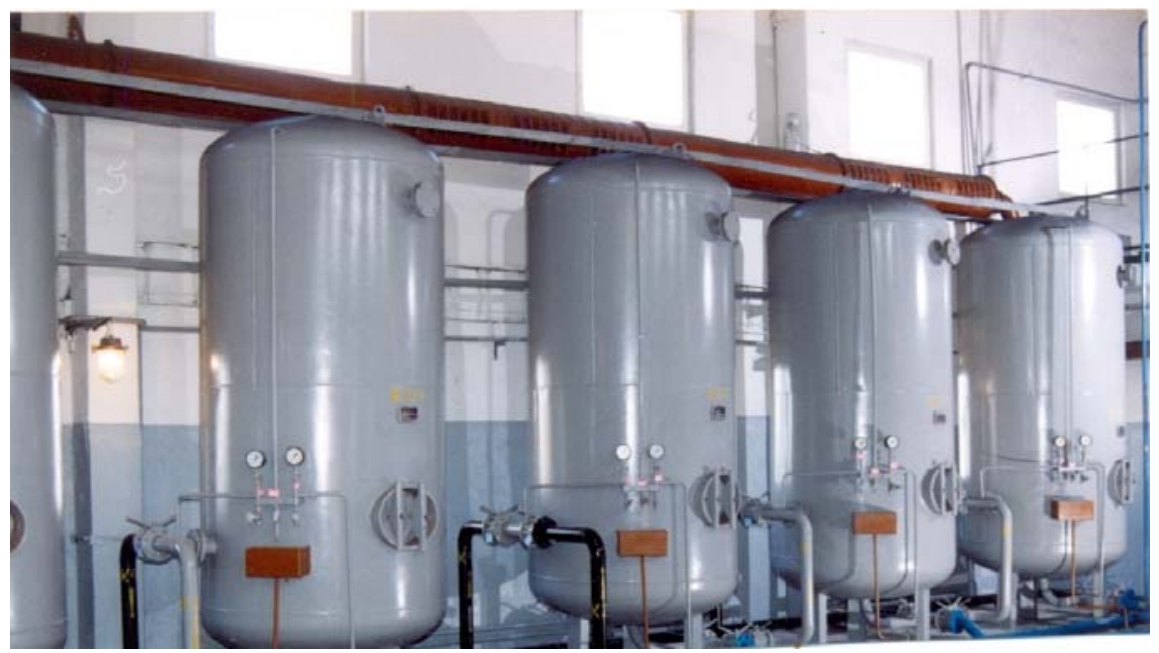

Fig. 2. Wastewater facilities for treatment of bus washing wastewater. 
In the course of observations on the operating treatment complexes it was revealed that quartz sand, shungite, basalt, zeolite, crushed expanded clay and anthracite crumb (fractional composition of $1-2 \mathrm{~mm}$ ) should be attributed to the most effective filtering materials meeting the requirements of the project. As a sorption material, fine-grained activated carbon of various grades is recommended: AG-3, AG-90, DAK, ART-1, BAU. High sorption capacity of activated carbons allows cleaning surface wastewater from fine sediment, dissolved oil products, salts of heavy metals, synthetic surface-active substances. Regular flushing of filtering and sorption materials with purified water accumulated in the collecting tank in countercurrent mode ensures effective removal of contaminants from the surfaces of their grains and from the interstitial space. Contaminated wash water is returned to the storage tank for further settling and cleaning on pressure filters.

To remove fallen sediment and petroleum products from storage tanks on the flowsheet a sand field provided with a device discharge of the supernatant liquid and afterespecially. After gravitational compression at the sand site under natural conditions the moisture content of the sludge is reduced to $50-60 \%$, which complies with the conditions of its export vehicles to special landfills for further disposal and recycling.

The supercharging suspension containing coarse and emulsified petrochemicals are subjected to gravity settling in the oil separator, while the watered mass of oil is accumulated and also exported to special enterprises for processing, and water enters the storage tank. The pumping equipment includes submersible pumps working in automatic mode and designed for transporting clarified water from storage tanks to pressure filters, backwashing and rinsing of load, as well as pumping sediment on the gravel pad.

The current environmental legislation regulates that in order to regulate the impact of economic activity on the environment, it is allowed both to determine the standards of permissible discharges of pollutants into water bodies (based on water quality standards of water bodies [4]), and the use of technological standards for large water users classified as category I, which have a significant negative impact on the environment [5]. In recent years, environmental legislation has been reformed. In accordance with the Federal law № 219-FZ for objects of the 1st category assigned to the scope of application of the best available technologies (BAT), technological standards will be established by a comprehensive environmental resolution based on indicators not exceeding the technological indicators of BAT [6,7]. This approach has been adopted in most developed countries.

Research JSC "MosvodokanalNIIproekt" showed that there is no universal surface runoff treatment technology due to the specific features of its formation and various degrees of pollution in urban territories of different functional purpose.

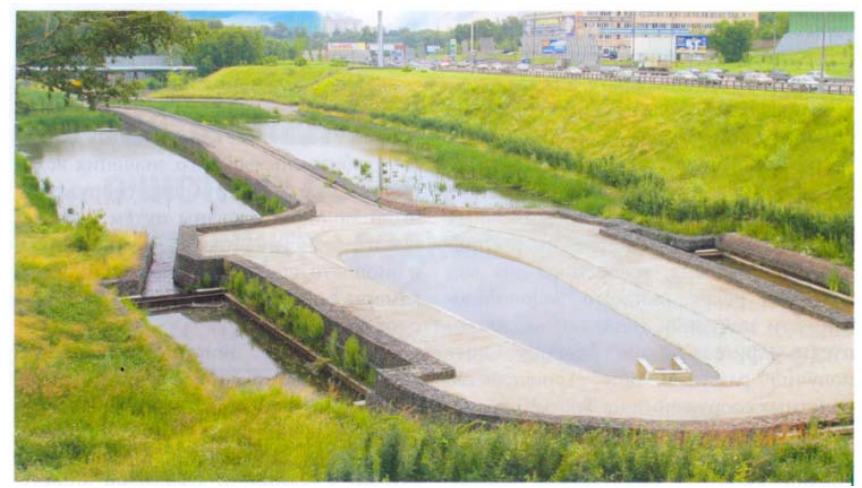

Fig. 3. Filtering water body (Moscow). 


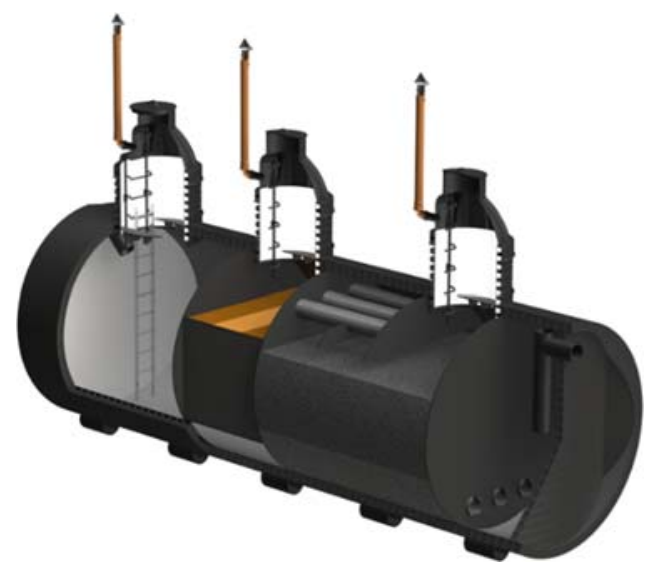

Fig. 4. Treatment facility (Polyplastic group).

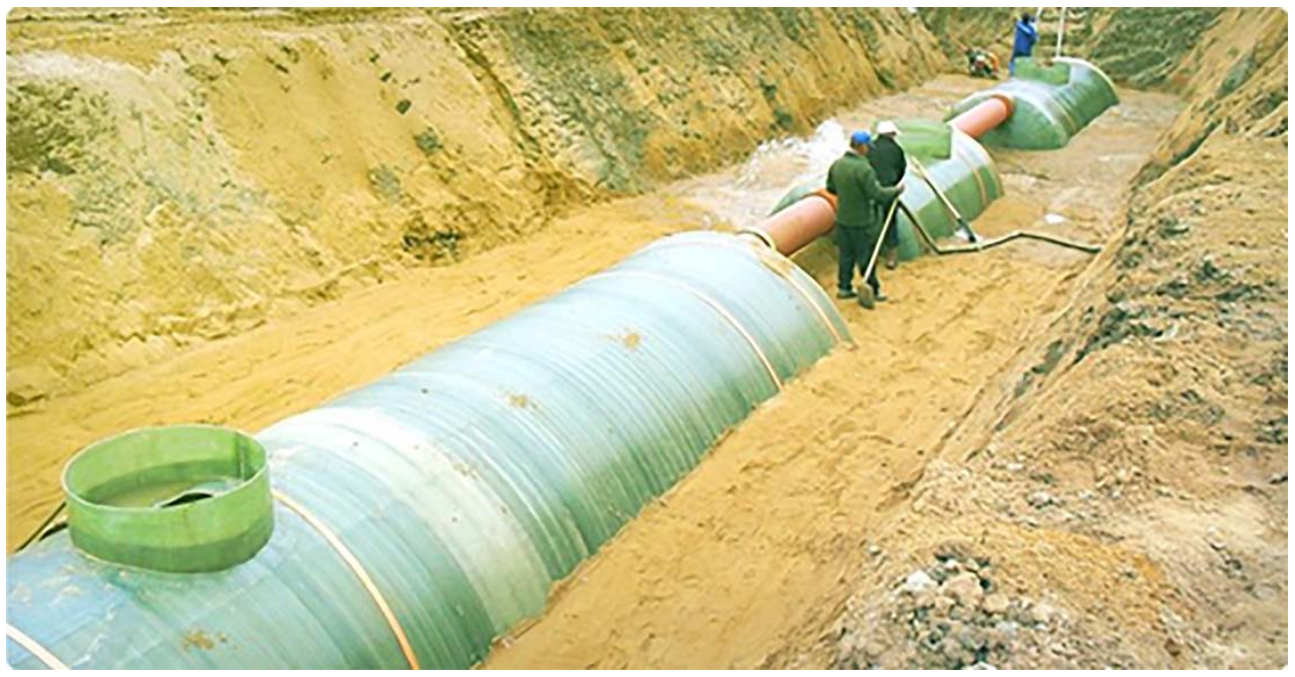

Fig. 5. Construction of rainwater treatment facility.

In residential areas, the composition of effluents-one in industrial parks, industrial zones-the other, with highways, gas stations - the third, etc. Great importance for the selection of treatment technology has surface wastewater and the number of effluents sent for cleaning. Along with proposals for the reconstruction and construction of group treatment facilities with the classical technology of surface wastewater treatment, it is necessary to consider the principle of decentralization of surface wastewater disposal and treatment with the extensive use of local treatment facilities providing requirements for the quality of treatment (Figures 3-5)

It is considered that flow-through structures required development of the rules of operation and, if necessary, fitting them to the storage facilities and biological treatment units. The use of flow structures at any particular facility should be accompanied by technological calculations according to the applicable design standards in the Russian Federation and guaranteed responsibility for ensuring the quality of surface runoff treatment in accordance with the declared efficiency and requirements for the quality of treated wastewater. 


\section{Conclusions}

1. At the present stage of reforming the regulation system of activities that have a negative impact on the environment the following technological issues for proposed wastewater treatment plants should be taken into account:

- The best available technologies in the field of wastewater treatment,

- Category and purpose of the water body,

- Hydraulic and chemical indicators of river water quality, formed under the influence of natural and man-made factors,

- Permissible anthropogenic load in order to preserve and restore the water body for safe water use of the population.

2. When determining engineering and technical measures for the construction and reconstruction of treatment facilities of surface runoff, it is necessary to take into account that for surface runoff there is no universal cleaning technology due to the specific features of its formation and the different degree of pollution of urban areas of various functional purposes. The quality and quantity of wastewater directed to treatment, accounting for the recommended BAT, is of great importance for the selection of surface wastewater treatment technology. All of this should be explored in the justification of scientific approaches and technologies for the design of treatment facilities of surface runoff, where it is necessary to develop the model a number of treatment facilities receiving runoff from urban areas with different functional purposes.

3. Along with proposals for the reconstruction and construction of wastewater treatment plants with the classical technology of surface water treatment, it is necessary to consider the principle of decentralization with the use of local treatment facilities.

\section{References}

1. General scheme of removal and treatment of surface runoff from Moscow for the period up to 2010 (Institute of MasterPlan of Moscow, 2001)

2. Target long-term program for the rehabilitation of water bodies of the city (JSC MosvodokanalNIIproekt, 2001)

3. G. Varyushina, N. Sveshnikova, Wat. Suppl. and Sanit Eng., 5, 35-39 (2017)

4. SanPiN 2.1.5.980-00 Hygienic requirements for the protection of surface waters (2001)

5. L. M.Vereshchagin, V. N. Shvetsov, Wat. Suppl. and Sanit Eng., 5, 29-34 (2017)

6. D. Danilovich, O. Chechevatov, Wat. Suppl. and Sanit Eng., 3, 18-23 (2015)

7. G. Varyushina, MATEC Web of Conf., 144, 05007 (2017) 